IJMMS 26:1 (2001) 21-34

PII. S0161171201003556

http://ijmms.hindawi.com

(c) Hindawi Publishing Corp.

\title{
PERISTALTIC VISCOELASTIC FLUID MOTION IN A TUBE
}

\author{
ELSAYED F. ELSHEHAWEY and AYMAN M. F. SOBH
}

(Received 24 June 1999)

\begin{abstract}
Peristaltic motion of viscoelastic incompressible fluid in an axisymmetric tube with a sinusoidal wave is studied theoretically in the case that the radius of the tube is small relative to the wavelength. Oldroyd flow has been considered in this study and the problem is formulated and analyzed using a perturbation expansion in terms of the variation of the wave number. This analysis can model the chyme movement in the small intestine by considering the chyme as an Oldroyd fluid. We found out that the pumping rate of Oldroyd fluid is less than that for a Newtonian fluid. Further, the effects of Reynolds number, Weissenberg number, amplitude ratio and wave number on the pressure rise and friction force have been discussed. It is found that the pressure rise does not depend on Weissenberg number at a certain value of flow rate. The results are studied for various values of the physical parameters of interest.
\end{abstract}

2000 Mathematics Subject Classification. 76Z05.

1. Introduction. A peristaltic pump is a device for pumping fluids, generally from a region of lower to higher pressure, by means of a contraction wave traveling along a tube-like structure. This traveling-wave phenomenon is referred to as (peristaltic). This phenomenon is now well known to physiologists to be one of the major mechanisms for fluid transport in many biological systems. The study of the mechanism of peristalsis, in both mechanical and physiological situations, has recently become the object of scientific research. Since the first investigation of Latham [6], several theoretical and experimental attempts have been made to understand peristaltic action in different situations. A review of much of the early literature is presented in an article by Jaffrin and Shapiro [5]. A summary of most of the experimental and theoretical investigations reported, with details of the geometry, fluid, Reynolds number, wavelength parameter, wave amplitude parameter, and wave shape has been given by Srivastava and Srivastava [11].

Most theoretical investigations have been carried out for Newtonian fluids, although it is known that most physiological fluids behave as non-Newtonian fluids. However, limited studies for non-Newtonian fluids have been made by Srivastava and Srivastava [12, 13], Srivastava [10], and Elshehawey and Mekheimer [4]. Bohme and Friedrich [1] have investigated peristaltic flow of second-order viscoelastic liquid assuming that the relevant Reynolds number is small enough to neglect inertial forces, and that the ratio of the wavelength and the channel height is large so that the pressure is constant over the cross-section. Peristaltic motion of a third-order fluid in a planar channel has been studied by Siddiqui and Schwarz [9], under the long-wavelength approximation assumption. El Misery et al. [2] studied the peristaltic motion of Carreau fluid in a channel. They developed the solution in a perturbation series in powers of Weissenberg 
number using long-wavelength approximation and also the same problem was studied by Elshehawey et al. [3] in the case of nonuniform channel.

The purpose of this paper is to study the peristaltic motion of Oldroyd fluid in a tube. This problem can model the movement of the chyme, which may be considered as Oldroyd fluid, through small intestine. In our analysis, we assumed that the velocity components, the pressure, the shearing stress, and the flow rate may be expanded in a regular perturbation series in the wave number. Expressions for pressure rise, velocity components and friction force were obtained in terms of the flow rate, the occlusion, the Reynolds number, the Weissenberg number, and the wave number.

2. Formulation and analysis. Consider the flow of an incompressible Oldroyd fluid in a circular tube of radius $a$. We assume an infinite wave train traveling with velocity $c$ along the wall. Taking $\bar{R}$ and $\bar{Z}$ as cylindrical coordinates, the geometry of the wall surface is

$$
\bar{h}(\bar{Z}, \bar{t})=a+b \sin \frac{2 \pi}{\lambda}(\bar{Z}-c \bar{t}),
$$

where $b$ is the wave amplitude, $\lambda$ is the wave length, and $\bar{Z}$ is the same direction of the wave propagation.

Choosing moving coordinates $(\bar{r}, \bar{z})$ (wave frame) which travel in the $\bar{Z}$-direction with the same speed as the wave, the unsteady flow in the laboratory frame $(\bar{R}, \bar{Z})$ can be treated as steady [8]. The coordinates frame are related through

$$
\bar{z}=\bar{Z}-c \bar{t}, \quad \bar{r}=\bar{R}, \quad \bar{w}=\bar{W}-c, \quad \bar{u}=\bar{U},
$$

where $\bar{U}, \bar{W}$ and $\bar{u}, \bar{w}$ are, respectively, the radial and the axial velocity components in the corresponding coordinate systems.

Equations of motion in the moving coordinates are

$$
\begin{gathered}
\frac{1}{\bar{r}} \frac{\partial}{\partial \bar{r}}(\bar{r} \bar{u})+\frac{\partial \bar{w}}{\partial \bar{z}}=0 \\
\rho\left(\bar{u} \frac{\partial \bar{u}}{\partial \bar{r}}+\bar{w} \frac{\partial \bar{u}}{\partial \bar{z}}\right)=-\frac{\partial \bar{p}}{\partial \bar{r}}-\left(\frac{1}{\bar{r}} \frac{\partial}{\partial \bar{r}}\left(\bar{r} \bar{\tau}_{11}\right)-\frac{\bar{\tau}_{22}}{\bar{r}}+\frac{\partial \bar{\tau}_{13}}{\partial \bar{z}}\right) \\
\rho\left(\bar{u} \frac{\partial \bar{w}}{\partial \bar{r}}+\bar{w} \frac{\partial \bar{w}}{\partial \bar{z}}\right)=-\frac{\partial \bar{p}}{\partial \bar{z}}-\left(\frac{1}{\bar{r}} \frac{\partial}{\partial \bar{r}}\left(\bar{r} \bar{\tau}_{13}\right)+\frac{\partial \bar{\tau}_{33}}{\partial \bar{z}}\right) .
\end{gathered}
$$

The constitutive equations of Oldroyd fluid are, [7],

$$
\begin{gathered}
\bar{\tau}_{11}+\Gamma\left[\bar{u} \frac{\partial \bar{\tau}_{11}}{\partial \bar{r}}+\bar{w} \frac{\partial \bar{\tau}_{11}}{\partial \bar{z}}-2 \bar{\tau}_{11} \frac{\partial \bar{u}}{\partial \bar{r}}-2 \bar{\tau}_{13} \frac{\partial \bar{u}}{\partial \bar{z}}\right]=-\mu \overline{\dot{\gamma}}_{11} \\
\bar{\tau}_{13}+\Gamma\left[\bar{u} \frac{\partial \bar{\tau}_{13}}{\partial \bar{r}}+\bar{w} \frac{\partial \bar{\tau}_{13}}{\partial \bar{z}}-\bar{\tau}_{33} \frac{\partial \bar{u}}{\partial \bar{z}}-\bar{\tau}_{11} \frac{\partial \bar{w}}{\partial \bar{r}}+\frac{\bar{u}}{\bar{r}} \bar{\tau}_{13}\right]=-\mu \overline{\dot{\gamma}}_{13} \\
\bar{\tau}_{22}+\Gamma\left[\bar{u} \frac{\partial \bar{\tau}_{22}}{\partial \bar{r}}+\bar{w} \frac{\partial \bar{\tau}_{22}}{\partial \bar{z}}-\frac{2 \bar{u}}{\bar{r}} \bar{\tau}_{22}\right]=-\mu \overline{\dot{\gamma}}_{22} \\
\bar{\tau}_{33}+\Gamma\left[\bar{u} \frac{\partial \bar{\tau}_{33}}{\partial \bar{r}}+\bar{w} \frac{\partial \bar{\tau}_{33}}{\partial \bar{z}}-2 \bar{\tau}_{33} \frac{\partial \bar{w}}{\partial \bar{z}}-2 \bar{\tau}_{13} \frac{\partial \bar{w}}{\partial \bar{r}}\right]=-\mu \overline{\dot{y}}_{33}
\end{gathered}
$$


where $\bar{p}$ is the pressure, $\bar{\tau}_{i j}, i, j=1,2,3$, are the components of the extra stress tensor, $\Gamma$ is the relaxation time, $\rho$ is the viscosity, and $\bar{\gamma}_{i j}, i, j=1,2,3$, are the components of strain-rate tensor and given by

$$
\overline{\dot{\gamma}}_{11}=2 \frac{\partial \bar{u}}{\partial \bar{r}}, \quad \overline{\dot{\gamma}}_{22}=2 \frac{\bar{u}}{\bar{r}}, \quad \overline{\dot{\gamma}}_{33}=2 \frac{\partial \bar{w}}{\partial \bar{z}}, \quad \overline{\dot{\gamma}}_{13}=\left(\frac{\partial \bar{u}}{\partial \bar{z}}+\frac{\partial \bar{w}}{\partial \bar{r}}\right)
$$

The boundary conditions are

$$
\frac{\partial \bar{w}}{\partial \bar{r}}=0, \quad \bar{u}=0 \quad \text { for } \bar{r}=0, \quad \bar{w}=-c, \quad \bar{u}=-c \frac{d \bar{h}}{d \bar{z}} \quad \text { for } \bar{r}=\bar{h}
$$

Introducing the nondimensional variables and parameters

$$
\begin{gathered}
z=\frac{\bar{z}}{\lambda}, \quad Z=\frac{\bar{Z}}{\lambda}, \quad r=\frac{\bar{r}}{a}, \quad R=\frac{\bar{R}}{a}, \quad t=\frac{c \bar{t}}{\lambda}, \quad p=\frac{a^{2} \bar{p}}{c \lambda \mu}, \\
u=\frac{\lambda \bar{u}}{a c}, \quad w=\frac{\bar{w}}{c}, \quad W=\frac{\bar{W}}{c}, \quad \delta=\frac{a}{\lambda}, \quad \tau_{i j}=\frac{a \bar{\tau}_{i j}}{c \mu}, \\
\dot{\gamma}_{i j}=\frac{a}{c} \overline{\dot{\gamma}}_{i j}, \quad \mathrm{Wi}=\frac{c \Gamma}{a}, \quad \operatorname{Re}=\frac{\rho c a}{\mu},
\end{gathered}
$$

where Wi is the Weissenberg number, $\delta$ is the wave number, Re is the Reynolds number, and $h=\bar{h} / a=1+(b / a) \sin 2 \pi z=1+\varphi \sin 2 \pi z, \varphi=b / a<1$, is the amplitude ratio, equations (2.3), (2.4), after using (2.5), become

$$
\begin{gathered}
\frac{1}{r} \frac{\partial}{\partial r}(r u)+\frac{\partial w}{\partial z}=0 \\
\operatorname{Re} \delta^{3}\left(u \frac{\partial u}{\partial r}+w \frac{\partial u}{\partial z}\right)=-\frac{\partial p}{\partial r}-\delta\left(\frac{1}{r} \frac{\partial}{\partial r}\left(r \tau_{11}\right)-\frac{\tau_{22}}{r}+\delta \frac{\partial \tau_{13}}{\partial z}\right) \\
\operatorname{Re} \delta\left(u \frac{\partial w}{\partial r}+w \frac{\partial w}{\partial z}\right)=-\frac{\partial p}{\partial z}-\left(\frac{1}{r} \frac{\partial}{\partial r}\left(r \tau_{13}\right)+\delta \frac{\partial \tau_{33}}{\partial z}\right) \\
\tau_{11}+\mathrm{Wi} \delta\left[u \frac{\partial \tau_{11}}{\partial r}+w \frac{\partial \tau_{11}}{\partial z}-2 \tau_{11} \frac{\partial u}{\partial r}-2 \delta \tau_{13} \frac{\partial u}{\partial z}\right]=-2 \delta\left(\frac{\partial u}{\partial r}\right) \\
\tau_{13}\left[\delta\left(u \frac{\partial \tau_{13}}{\partial r}+w \frac{\partial \tau_{13}}{\partial z}-\delta \tau_{33} \frac{\partial u}{\partial z}+\frac{u}{r} \tau_{13}\right)-\tau_{11} \frac{\partial w}{\partial r}\right]=-\left(\delta^{2} \frac{\partial u}{\partial z}+\frac{\partial w}{\partial r}\right) \\
\tau_{22}+\mathrm{Wi} \delta\left[u \frac{\partial \tau_{22}}{\partial r}+w \frac{\partial \tau_{22}}{\partial z}-\frac{2 u}{r} \tau_{22}\right]=-2 \delta \frac{u}{r} \\
\text { Wi }\left[\delta\left(u \frac{\partial \tau_{33}}{\partial r}+w \frac{\partial \tau_{33}}{\partial z}-2 \tau_{33} \frac{\partial w}{\partial z}\right)-2 \tau_{13} \frac{\partial w}{\partial r}\right]=-2 \delta\left(\frac{\partial w}{\partial z}\right)
\end{gathered}
$$

The nondimensional boundary conditions are

$$
\frac{\partial w}{\partial r}=0, \quad u=0 \quad \text { for } r=0, \quad w=-1, \quad u=-\frac{d h}{d z} \text { for } r=h
$$


Eliminating the pressure from (2.9) and (2.10), we obtain

$$
\begin{aligned}
\operatorname{Re} \delta\left[\delta^{2}\right. & \left.\frac{\partial}{\partial z}\left(u \frac{\partial u}{\partial r}+w \frac{\partial u}{\partial z}\right)-\frac{\partial}{\partial r}\left(u \frac{\partial w}{\partial r}+w \frac{\partial w}{\partial z}\right)\right] \\
= & \frac{\partial}{\partial r}\left(\frac{1}{r} \frac{\partial}{\partial r}\left(r \tau_{13}\right)+\delta \frac{\partial \tau_{33}}{\partial z}\right)-\delta \frac{\partial}{\partial z}\left(\frac{1}{r} \frac{\partial}{\partial r}\left(r \tau_{11}\right)-\frac{\tau_{22}}{r}+\delta \frac{\partial \tau_{13}}{\partial z}\right) .
\end{aligned}
$$

3. Rate of volume flow. The instantaneous volume flow rate in the fixed frame is given by

$$
Q=2 \pi \int_{0}^{\bar{h}} \bar{W} \bar{R} d \bar{R}
$$

where $\bar{h}$ is a function of $\bar{Z}$ and $\bar{t}$.

The rate of volume flow in the moving frame (wave frame) is given by

$$
\bar{q}=2 \pi \int_{0}^{\bar{h}} \bar{w} \bar{r} d \bar{r}
$$

where $\bar{h}$ is a function of $\bar{z}$.

Using (3.2), one finds that the two rates of volume flow are related by

$$
Q=\bar{q}+\pi c \bar{h}^{2}
$$

The time-mean flow over a period $T=\lambda / c$ at a fixed position $\bar{Z}$ is defined as

$$
\bar{Q}=\frac{1}{T} \int_{0}^{T} Q d \bar{t}
$$

which can be written, using (2.1) and (3.3), as

$$
\bar{Q}=\bar{q}+\pi c a^{2}\left(1+\frac{\varphi^{2}}{2}\right) .
$$

Defining the dimensionless time-mean flows $\theta$ and $F$ in the fixed and wave frame, respectively, as

$$
\theta=\frac{\bar{Q}}{\pi c a^{2}}, \quad F=\frac{\bar{q}}{\pi c a^{2}},
$$

then making use of (3.6), equation (3.5) can be rewritten as

$$
\theta=F+1+\frac{\varphi^{2}}{2}
$$

where

$$
F=2 \int_{0}^{h(z)} r w d r
$$


4. Perturbation solution. Beginning by expanding the following quantities in a power series of the small parameter $\delta$ as follows:

$$
\begin{aligned}
& u=u_{0}+\delta u_{1}+\delta^{2} u_{2}+O\left(\delta^{3}\right), \quad w=w_{0}+\delta w_{1}+\delta^{2} w_{2}+O\left(\delta^{3}\right), \\
& \frac{\partial p}{\partial z}=\frac{\partial p_{0}}{\partial z}+\delta \frac{\partial p_{1}}{\partial z}+\delta^{2} \frac{\partial p_{2}}{\partial z}+O\left(\delta^{3}\right), \quad \tau_{11}=\tau_{11}^{(0)}+\delta \tau_{11}^{(1)}+\delta^{2} \tau_{11}^{(2)}+O\left(\delta^{3}\right), \\
& \tau_{13}=\tau_{13}^{(0)}+\delta \tau_{13}^{(1)}+\delta^{2} \tau_{13}^{(2)}+O\left(\delta^{3}\right), \quad \tau_{22}=\tau_{11}^{(0)}+\delta \tau_{22}^{(1)}+\delta^{2} \tau_{22}^{(2)}+O\left(\delta^{3}\right), \\
& \tau_{33}=\tau_{33}^{(0)}+\delta \tau_{33}^{(1)}+\delta^{2} \tau_{33}^{(2)}+O\left(\delta^{3}\right), \quad F=F_{0}+\delta F_{1}+\delta^{2} F_{2}+O\left(\delta^{3}\right),
\end{aligned}
$$

then using the perturbation expansions (4.1) in equations (2.8), (2.10), (2.11), (2.12), (2.13), (2.14), (2.15), (2.16), and (3.8) and collecting terms of like powers of $\delta$, we obtain three sets of coupled linear differential equations with their corresponding boundary conditions in $u_{0}, w_{0}, u_{1}, w_{1}$, and $u_{2}, w_{2}$ for the first three powers of $\delta$. The first set of differential equations in $u_{0}, w_{0}$, subject to the corresponding boundary conditions, yields the following classical Poiseuille flow:

$$
\begin{gathered}
w_{0}=c_{1}+c_{2} r^{2}, \\
u_{0}=-\frac{c_{1}^{\prime}}{2} r-\frac{c_{2}^{\prime}}{4} r^{3},
\end{gathered}
$$

where

$$
c_{1}=1+\frac{2 F_{0}}{h^{2}}, \quad c_{2}=-2\left(\frac{F_{0}+h^{2}}{h^{4}}\right) .
$$

On substituting the zeroth-order solution (4.2) in the second set of differential equations and using its corresponding boundary conditions, the first-order solution can be obtained in the form

$$
\begin{gathered}
w_{1}=c_{3}+c_{4} r^{2}+c_{5} r^{4}+c_{6} r^{6}, \\
u_{1}=-\frac{c_{3}^{\prime}}{2} r-\frac{c_{4}^{\prime}}{4} r^{3}-\frac{c_{5}^{\prime}}{6} r^{5}-\frac{c_{6}^{\prime}}{8} r^{7},
\end{gathered}
$$

where

$$
\begin{aligned}
c_{3}=\frac{2 F_{1}}{h^{2}}+\operatorname{Re}\left(\frac{c_{1} c_{2}^{\prime} h^{4}}{48}+\frac{c_{2} c_{2}^{\prime} h^{6}}{144}\right), & c_{4}=-\frac{2 F_{1}}{h^{4}}-\operatorname{Re}\left(\frac{c_{1} c_{2}^{\prime} h^{2}}{12}+\frac{c_{2} c_{2}^{\prime} h^{4}}{48}\right), \\
c_{5}=\operatorname{Re}\left(\frac{c_{1} c_{2}^{\prime}}{16}\right), & c_{6}=\operatorname{Re}\left(\frac{c_{2} c_{2}^{\prime}}{72}\right) .
\end{aligned}
$$

We now solve the second-order system. Using the zeroth-order and the first-order solutions in the third set of differential equations and using the boundary conditions, we obtain

$$
w_{2}=b_{1}+b_{2} r^{2}+b_{3} r^{4}+b_{4} r^{6}+b_{5} r^{8}+b_{6} r^{10}
$$

where

$$
\begin{aligned}
b_{1}= & \frac{2 F_{2}}{h^{2}}-\frac{c_{2}^{\prime \prime} h^{4}}{24}+\operatorname{Re}\left(\frac{h^{4} a_{1}}{48}+\frac{h^{6} a_{2}}{72}+\frac{3 h^{8} a_{3}}{320}+\frac{h^{10} a_{4}}{150}\right) \\
& +\mathrm{Wi}\left(\frac{h^{4} a_{5}}{12}+\frac{h^{6} a_{6}}{12}+\frac{3 h^{8} a_{7}}{40}\right)-\mathrm{Wi}^{2}\left(\frac{h^{4} a_{8}}{12}+\frac{h^{6} a_{9}}{12}\right),
\end{aligned}
$$




$$
\begin{aligned}
b_{2}= & -\frac{2 F_{2}}{h^{4}}+\frac{c_{2}^{\prime \prime} h^{2}}{6}-\operatorname{Re}\left(\frac{h^{2} a_{1}}{12}+\frac{h^{4} a_{2}}{24}+\frac{h^{6} a_{3}}{40}+\frac{h^{8} a_{4}}{60}\right) \\
& -W\left(\frac{h^{2} a_{5}}{3}+\frac{h^{4} a_{6}}{4}+\frac{h^{6} a_{7}}{5}\right)+\mathrm{Wi}^{2}\left(\frac{h^{2} a_{8}}{3}+\frac{h^{4} a_{9}}{4}\right), \\
b_{3}= & -\frac{c_{2}^{\prime \prime}}{8}+\frac{1}{16}\left(\operatorname{Re} a_{1}+4 \mathrm{Wi} a_{5}-4 \mathrm{Wi}^{2} a_{8}\right), \\
b_{4}= & \frac{1}{36}\left(\operatorname{Re} a_{2}+6 \mathrm{Wi} a_{6}-6 \mathrm{Wi}^{2} a_{9}\right), \\
b_{5}= & \frac{1}{64}\left(\operatorname{Re} a_{3}+8 \mathrm{Wi} a_{7}\right), \quad b_{6}=\frac{\operatorname{Re} a_{4}}{100}, \\
a_{1}= & \left(c_{3} c_{2}^{\prime}+c_{1} c_{4}^{\prime}\right), \quad a_{2}=\left(c_{1} c_{5}^{\prime}-c_{5} c_{1}^{\prime}+\frac{c_{4} c_{2}^{\prime}}{2}+\frac{c_{2} c_{4}^{\prime}}{4}\right), \\
a_{3}= & \left(c_{1} c_{6}^{\prime}-2 c_{6} c_{1}^{\prime}+\frac{2 c_{2} c_{5}^{\prime}}{3}\right), \quad a_{4}=\left(\frac{3 c_{2} c_{6}^{\prime}}{4}-\frac{c_{6} c_{2}^{\prime}}{2}\right), \quad a_{5}=4\left(c_{1} c_{5}^{\prime}-c_{5} c_{1}^{\prime}\right), \\
a_{6}= & \left(6 c_{1} c_{6}^{\prime}-12 c_{6} c_{1}^{\prime}+\frac{4 c_{2} c_{5}^{\prime}}{3}-\frac{4 c_{5} c_{2}^{\prime}}{3}\right), \quad a_{7}=\left(3 c_{2} c_{6}^{\prime}-4 c_{6} c_{2}^{\prime}\right), \\
a_{8}= & \left(3 c_{2} c_{1}^{\prime} c_{2}^{\prime}-3 c_{1} c_{2}^{\prime 2}+3 c_{1} c_{2} c_{2}^{\prime \prime}\right), \quad a_{9}=\left(c_{2}^{2} c_{2}^{\prime \prime}-c_{2} c_{2}^{\prime 2}\right) .
\end{aligned}
$$

At this order, the perturbation solution for the axial velocity can be, using (4.2a), (4.4a), and (4.6), written as

$$
\begin{aligned}
w= & c_{1}+c_{2} r^{2}+\delta\left(c_{3}+c_{4} r^{2}+c_{5} r^{4}+c_{6} r^{6}\right) \\
& +\delta^{2}\left(b_{1}+b_{2} r^{2}+b_{3} r^{4}+b_{4} r^{6}+b_{5} r^{8}+b_{6} r^{10}\right) .
\end{aligned}
$$

A close look at (4.8) reveals that the axial velocity is affected by the wave number, the Reynolds number and the viscoelastic parameter (Weissenberg number).

5. Pressure gradient. An expression for the pressure gradient, $\partial p / \partial z$, can be obtained by substituting (4.1) into the dimensionless equation of motion (2.10) and equating the coefficients of like powers of $\delta$, we obtain three sets of partial differential equations for $\partial p_{0} / \partial z, \partial p_{1} / \partial z$, and $\partial p_{2} / \partial z$. Using this form of $\partial p / \partial z$, the pressure rise and the friction force per wavelength can be obtained.

The nondimensional pressure rise and the nondimensional friction force per wavelength are defined, respectively, as

$$
\Delta p_{\lambda}=\int_{0}^{1} \frac{d p}{d z} d z, \quad F_{\lambda}=\int_{0}^{1} h^{2}\left(-\frac{d p}{d z}\right) d z .
$$

Since $\partial p / \partial z$ is periodic in $z$, the pressure rise and the friction force per wavelength in the longitudinal direction are independent of $r$, [5]. Accordingly, the integrals in (5.1) can be evaluated on the axis at $r=0$. Further, the pressure rise and friction force can be expanded as a power series in $\delta$ as

$$
\begin{aligned}
\Delta p_{\lambda} & =\Delta p_{\lambda 0}+\delta \Delta p_{\lambda 1}+\delta^{2} \Delta p_{\lambda 2}+\cdots, \\
F_{\lambda} & =F_{\lambda 0}+\delta F_{\lambda 1}+\delta^{2} F_{\lambda 2}+\cdots,
\end{aligned}
$$


where

$$
\begin{gathered}
\Delta p_{\lambda 0}=\int_{0}^{1} \frac{d p_{0}}{d z} d z, \quad \Delta p_{\lambda 1}=\int_{0}^{1} \frac{d p_{1}}{d z} d z, \quad \Delta p_{\lambda 2}=\int_{0}^{1} \frac{d p_{2}}{d z} d z \\
F_{\lambda 0}=\int_{0}^{1} h^{2}\left(-\frac{d p_{0}}{d z}\right) d z, \quad F_{\lambda 1}=\int_{0}^{1} h^{2}\left(-\frac{d p_{1}}{d z}\right) d z, \quad F_{\lambda 2}=\int_{0}^{1} h^{2}\left(-\frac{d p_{2}}{d z}\right) d z .
\end{gathered}
$$

We now use the zeroth-, first-, and second-order terms for the pressure gradient in (5.3), integrating from 0 to 1 , then substituting in (5.2) we obtain

$$
\begin{aligned}
& \Delta p_{\lambda}^{(2)}=-8 F^{(2)} I_{4}-8 I_{2} \\
&+\delta^{2}\left[-\frac{32 \pi^{2}}{3}\left(3\left(\varphi^{2}-1\right) I_{2}+5 I_{1}-2\right)\right. \\
&+F^{(2)}\{-\left.\frac{16 \pi^{2}}{3}\left(11\left(\varphi^{2}-1\right) I_{4}+21 I_{3}-10 I_{2}\right)\right\} \\
&+\operatorname{Re}^{2}\left\{\left(F^{(2)}\right)^{3}\left[\frac{22 \pi^{2}}{135}\left(11\left(\varphi^{2}-1\right) I_{6}+19 I_{5}-8 I_{4}\right)\right]\right. \\
&+\left(F^{(2)}\right)^{2}\left[\frac{\pi^{2}}{135}\left(115\left(\varphi^{2}-1\right) I_{4}+137 I_{3}-22 I_{2}\right)\right] \\
&-\left.\frac{\pi^{2} F^{(2)}}{135}\left(41\left(\varphi^{2}-1\right) I_{2}+121 I_{1}-80\right)-\frac{\pi^{2} \varphi^{2}}{27}\right\} \\
&-\operatorname{ReWi}^{2}\left\{\left(F^{(2)}\right)^{3}\left[\frac{32 \pi^{2}}{5}\left(7\left(\varphi^{2}-1\right) I_{8}+13 I_{7}-6 I_{6}\right)\right]\right. \\
&+\left(F^{(2)}\right)^{2}\left[\frac{32 \pi^{2}}{15}\left(19\left(\varphi^{2}-1\right) I_{6}+33 I_{5}-14 I_{4}\right)\right] \\
&+F^{(2)}\left[128 \pi^{2}\left(6\left(\varphi^{2}-1\right) I_{6}+11 I_{5}-5 I_{4}\right)\right] \\
&+F^{(2)}\left[\frac{8 \pi^{2}}{15}\left(11\left(\varphi^{2}-1\right) I_{4}+13 I_{3}-2 I_{2}\right)\right] \\
&\left.+\frac{8 \pi^{2}}{15}\left(\left(F^{2}-1\right) I_{2}+3 I_{1}-2\right)\right\}
\end{aligned}
$$




$$
\begin{aligned}
& F_{\lambda}^{(2)}=8 F^{(2)} I_{2} \\
& +8+\delta^{2}\left[\frac{64 \pi^{2} \varphi^{2}}{3}+F^{(2)}\left\{\frac{16 \pi^{2}}{3}\left(11\left(\varphi^{2}-1\right) I_{2}+21 I_{1}-10\right)\right\}\right. \\
& -\operatorname{Re}^{2}\left\{\left(F^{(2)}\right)^{3}\left[\frac{22 \pi^{2}}{135}\left(11\left(\varphi^{2}-1\right) I_{4}+19 I_{3}-8 I_{2}\right)\right]\right. \\
& +\frac{\pi^{2}\left(F^{(2)}\right)^{2}}{135}\left(115\left(\varphi^{2}-1\right) I_{2}+137 I_{1}-22\right) \\
& \left.-\frac{\pi^{2} \varphi^{2} F^{(2)}}{135}-\frac{\pi^{2} \varphi^{2}\left(\varphi^{2}+4\right)}{1080}\right\} \\
& +\operatorname{ReWi}\left\{\left(F^{(2)}\right)^{3}\left[\frac{32 \pi^{2}}{5}\left(7\left(\varphi^{2}-1\right) I_{6}+13 I_{5}-6 I_{4}\right)\right]\right. \\
& +\left(F^{(2)}\right)^{2}\left[\frac{32 \pi^{2}}{15}\left(19\left(\varphi^{2}-1\right) I_{4}+33 I_{3}-14 I_{2}\right)\right] \\
& \left.+\left(F^{(2)}\right)^{2}\left[\frac{8 \pi^{2}}{15}\left(11\left(\varphi^{2}-1\right) I_{2}+13 I_{1}-2\right)\right]\right\} \\
& +\mathrm{Wi}^{2}\left\{\left(F^{(2)}\right)^{3}\left[128 \pi^{2}\left(19\left(\varphi^{2}-1\right) I_{8}+35 I_{7}-16 I_{6}\right)\right]\right. \\
& +\left(F^{(2)}\right)^{2}\left[64 \pi^{2}\left(37\left(\varphi^{2}-1\right) I_{6}+67 I_{5}-30 I_{4}\right)\right] \\
& +F^{(2)}\left[128 \pi^{2}\left(6\left(\varphi^{2}-1\right) I_{4}+11 I_{3}-5 I_{2}\right)\right] \\
& \left.\left.+128 \pi^{2}\left(\left(\varphi^{2}-1\right) I_{2}+2 I_{1}-1\right)\right\}\right]
\end{aligned}
$$

where

$$
I_{n}=\int_{0}^{1} \frac{1}{h^{n}} d z, \quad n=1,2, \ldots, 10
$$

Here, we used the relation

$$
F_{0}=F^{(2)}-\delta F_{1}-\delta^{2} F_{2},
$$


and $\Delta p_{\lambda}^{(2)}, F_{\lambda}^{(2)}$, and $F^{(2)}$ are, respectively, the pressure rise, the friction force and the flow rate in the wave frame to the second order in $\delta$, and where

$$
\begin{gathered}
I_{1}=\frac{1}{\left(1-\varphi^{2}\right)^{1 / 2}}, \quad I_{2}=\frac{1}{\left(1-\varphi^{2}\right)^{3 / 2}}, \quad I_{3}=\frac{\left(1+\varphi^{2} / 2\right)}{\left(1-\varphi^{2}\right)^{5 / 2}}, \quad I_{4}=\frac{\left(1+3 \varphi^{2} / 2\right)}{\left(1-\varphi^{2}\right)^{7 / 2}}, \\
I_{n}=\frac{1}{\left(1-\varphi^{2}\right)}\left[\left(\frac{2 n-3}{n-1}\right) I_{n-1}-\left(\frac{n-2}{n-1}\right) I_{n-2}\right], \quad n>4 .
\end{gathered}
$$

The substitution of (5.8) into (5.4) and (5.5) yields

$$
\begin{aligned}
& \Delta p_{\lambda}^{(2)}=-\frac{8\left(1+3 \varphi^{2} / 2\right)}{\left(1-\varphi^{2}\right)^{7 / 2}}\left(\theta^{(2)}-1-\frac{\varphi^{2}}{2}\right)-\frac{8}{\left(1-\varphi^{2}\right)^{3 / 2}} \\
&+\delta^{2}\left[-\frac{64 \pi^{2} \varphi^{2}}{3\left(1-\varphi^{2}\right)^{5 / 2}}\left(\theta^{(2)}-1-\frac{\varphi^{2}}{2}\right)-\frac{64 \pi^{2}}{3}\left\{\frac{1}{\left(1-\varphi^{2}\right)^{1 / 2}}-1\right\}\right. \\
&+\operatorname{ReWi}\left\{\frac{8 \pi^{2} \varphi^{2}\left(4-\varphi^{2}-3 \varphi^{4}\right)\left(\theta^{(2)}-1-\varphi^{2} / 2\right)^{2}}{5\left(1-\varphi^{2}\right)^{11 / 2}}\right. \\
&+\operatorname{Re}^{2}\left\{\frac{11 \pi^{2} \varphi^{2}\left(3 \varphi^{4}+\varphi^{2}-4\right)\left(\theta^{(2)}-1-\varphi^{2} / 2\right)^{3}}{135\left(1-\varphi^{2}\right)^{11 / 2}}\right.\left.+\frac{64 \pi^{2} \varphi^{2}\left(\theta^{(2)}-1-\varphi^{2} / 2\right)}{15\left(1-\varphi^{2}\right)^{5 / 2}}-\frac{16 \pi^{2}}{15}\left(1-\frac{1}{\left(1-\varphi^{2}\right)^{1 / 2}}\right)\right\} \\
&+\frac{82 \pi^{2} \varphi^{2}\left(\theta^{(2)}-1-\varphi^{2} / 2\right)^{2}}{135\left(1-\varphi^{2}\right)^{5 / 2}} \\
&+\operatorname{Wi}^{2}\left\{\frac{8 \pi \varphi^{2}\left(35 \varphi^{6}+280 \varphi^{4}+336 \varphi^{2}+64\right)}{\left(1-\varphi^{2}\right)^{17 / 2}}\left(\theta^{(2)}-1-\frac{\varphi^{2}}{2}\right)\right. \\
&+
\end{aligned}
$$


and

$$
\begin{aligned}
& F_{\lambda}^{(2)}=\frac{8\left(\theta^{(2)}-1-\varphi^{2} / 2\right)}{\left(1-\varphi^{2}\right)^{3 / 2}} \\
& +8+\delta^{2}\left[\frac{160 \pi^{2}}{3}\left(\frac{1}{\left(1-\varphi^{2}\right)^{1 / 2}}-1\right)\left(\theta^{(2)}-1-\frac{\varphi^{2}}{2}\right)+\frac{64 \pi^{2} \varphi^{2}}{3}\right. \\
& +\operatorname{ReWi}\left\{\frac{8 \pi^{2} \varphi^{2}\left(4-\varphi^{2}-3 \varphi^{4}\right)\left(\theta^{(2)}-1-\varphi^{2} / 2\right)^{3}}{5\left(1-\varphi^{2}\right)^{11 / 2}}\right. \\
& +\frac{64 \pi^{2} \varphi^{2}\left(\theta^{(2)}-1-\varphi^{2} / 2\right)^{2}}{15\left(1-\varphi^{2}\right)^{5 / 2}} \\
& \left.-\frac{16 \pi^{2}}{15}\left(1-\frac{1}{\left(1-\varphi^{2}\right)^{1 / 2}}\right)\left(\theta^{(2)}-1-\frac{\varphi^{2}}{2}\right)\right\} \\
& +\operatorname{Re}^{2}\left\{-\frac{22 \pi^{2} \varphi^{2}\left(\theta^{(2)}-1-\varphi^{2} / 2\right)^{2}}{135\left(1-\varphi^{2}\right)^{5 / 2}}\right. \\
& +\frac{22 \pi^{2}}{15}\left(1-\frac{1}{\left(1-\varphi^{2}\right)^{1 / 2}}\right)\left(\theta^{(2)}-1-\frac{\varphi^{2}}{2}\right) \\
& \left.+\frac{\pi^{2} \varphi^{2}}{135}\left(\theta^{(2)}-1-\frac{\varphi^{2}}{2}\right)+\frac{\pi^{2} \varphi^{2}\left(\varphi^{2}+4\right)}{1080}\right\} \\
& -\mathrm{Wi}^{2}\left\{\frac{16 \pi^{2} \varphi^{2}\left(5 \varphi^{4}+20 \varphi^{2}+8\right)}{\left(1-\varphi^{2}\right)^{13 / 2}}\left(\frac{\theta^{(2)}-1-\varphi^{2}}{2}\right)^{3}\right. \\
& +\frac{16 \pi^{2} \varphi^{2}\left(3 \varphi^{4}+\varphi^{2}-4\right)}{\left(1-\varphi^{2}\right)^{11 / 2}}\left(\theta^{(2)}-1-\frac{\varphi^{2}}{2}\right)^{2} \\
& \left.\left.-\frac{192 \pi^{2} \varphi^{2}}{\left(1-\varphi^{2}\right)^{5 / 2}}\left(\theta^{(2)}-1-\frac{\varphi^{2}}{2}\right)+128 \pi^{2}\left(1-\frac{1}{\left(1-\varphi^{2}\right)^{1 / 2}}\right)\right\}\right] \text {, }
\end{aligned}
$$

where

$$
\theta^{(2)}=F^{(2)}+1+\frac{\varphi^{2}}{2}
$$

6. Results and conclusion. It is clear that our results calculate the velocity, the pressure rise and the friction force without restrictions on the amplitude ratio, the Reynolds number and the Weissenberg number but we used a small wave number. Further, the results extend the work of Shapiro et al. [8] as well as it include the effect of Weissenberg number Wi.

In Figure 6.1, the dimensionless pressure rise $\left(\Delta p_{\lambda}\right)^{(2)}$ is graphed versus the dimensionless flow rate $\theta^{(2)}$ for different values of Weissenberg number (Wi $=0,0.04,0.08$ ) at wave number $\delta=0.156$ and Reynolds number $\operatorname{Re}=0.1$, for both cases $(\varphi=0.35$ and $\varphi=0.6$ ). As shown, for $\varphi=0.35$, the effect of Weissenberg number is very small and the three curves coincide. But for $\varphi=0.6$, the effect of Weissenberg number is 


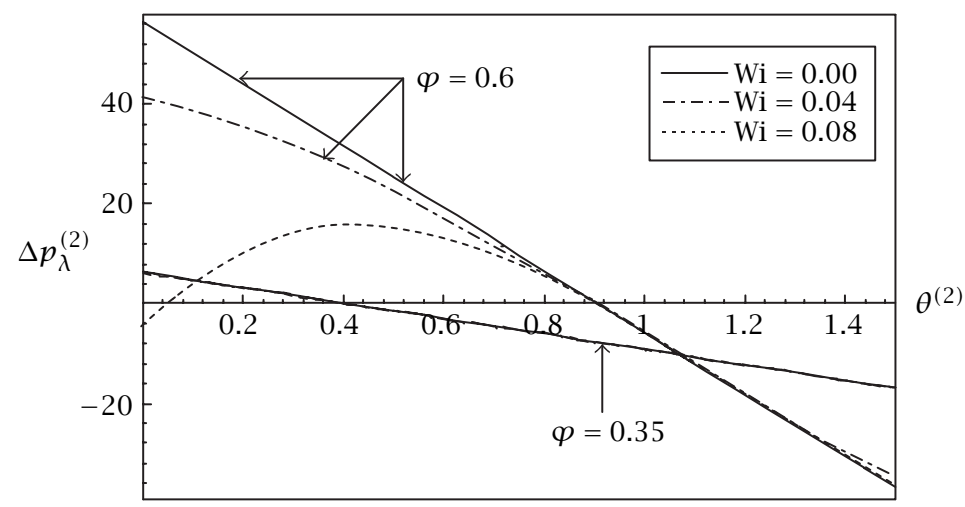

FIGURE 6.1. The pressure rise versus flow rate at $\mathrm{Re}=0.1, \delta=0.156$, and $\varphi=0.35,0.6$.

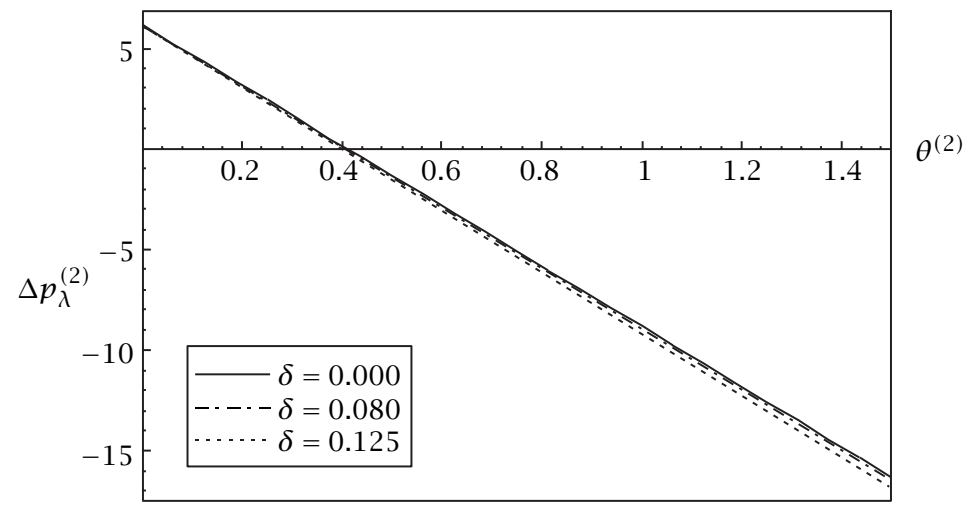

FIGURE 6.2. The pressure rise versus flow rate at $\mathrm{Re}=0.1, \mathrm{Wi}=0.08$, and $\varphi=0.35$.

very clear and show that the pumping rate of Oldroyd fluid is less than that for a Newtonian having a shear viscosity the same as Oldroyd fluid and the pressure rise decreases with increasing Weissenberg number. Further, it is clear that the pressure rise is independent on Weissenberg number at a certain value of flow rate and the peristaltic pumping, where $\left(\Delta p_{\lambda}\right)^{(2)}>0$ and $\theta^{(2)}>0$, occur at $0 \leq \theta^{(2)} \leq 0.9$ and the augmented pumping, where $\left(\Delta p_{\lambda}\right)^{(2)}<0$ and $\theta^{(2)}>0$, occur at $0.9 \leq \theta^{(2)} \leq 1.5$, for $\varphi=0.6$. The linear relation for a Newtonian fluid is obvious in (5.9), with $\mathrm{Wi}=0$ and $\delta=0$. Figures 6.2 and 6.3 show the effect of the wave number $\delta$ on the pressure rise at $\mathrm{Re}=0.1, \mathrm{Wi}=0.08$, and $\varphi=0.35,0.6$, respectively. Figure 6.2 reveals that, for $\varphi=0.35$, an increase in the wave number yields a slight increase in the magnitude of the pressure rise but for $\varphi=0.6$, the effect of the wave number $\delta$ is very clear and the pressure rise decreases as wave number increases as shown in Figure 6.3.

Shown in Figure 6.4 the dimensionless pressure rise versus flow rate at $\mathrm{Wi}=0, \delta=$ $0.02, \varphi=0.6$, and $\operatorname{Re}=0,50,100$. The results reveal that the magnitude of the pressure rise increases with increasing Reynolds number. 


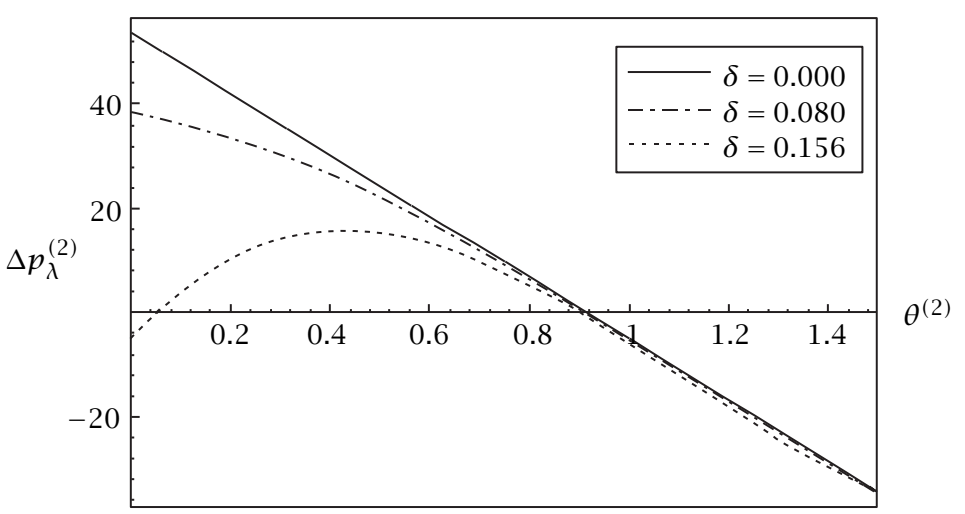

FigURE 6.3. The pressure rise versus flow rate at $\mathrm{Re}=0.1, \mathrm{Wi}=0.08$, and $\varphi=0.6$.

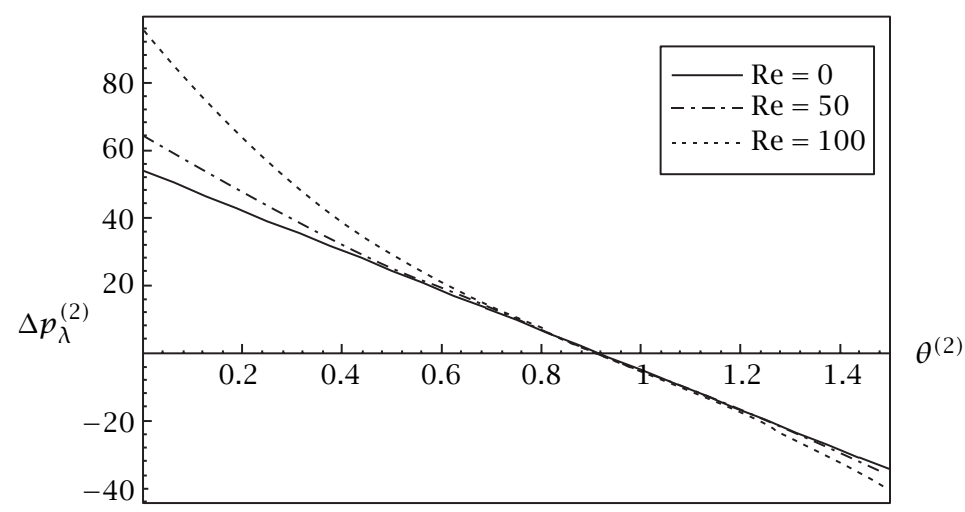

FIGURE 6.4. The pressure rise versus flow rate at $\mathrm{Wi}=0, \delta=0.02$, and $\varphi=0.6$.

The dimensionless friction force is plotted versus flow rate in Figures 6.5, 6.6, 6.7, and 6.8. Figure 6.5 shows the friction force versus flow rate at $\delta=0.156, \operatorname{Re}=0.1$, and $\mathrm{Wi}=0,0.04,0.08$ in both cases $\varphi=0.35$ and $\varphi=0.6$. It is shown that the friction force is independent on Weissenberg number at $\varphi=0.35$ but its magnitude decreases with increasing Weissenberg number at $\varphi=0.6$ and it does not depend on Weissenberg number at a certain value of flow rate in this case. Shown in Figures 6.6 and 6.7 the effect of wave number $\delta$ on the friction force at $\varphi=0.35$ and $\varphi=0.6$, respectively. We notice from Figure 6.6 that the magnitude of the friction force increases with increasing the wave number and it is independent on wave number at a certain value of flow rate. This result is different at $\varphi=0.6$ as shown in Figure 6.7. Finally, the friction force is displayed versus flow rate in Figure 6.8 at $\mathrm{Wi}=0, \delta=0.02$, and $\varphi=0.6$, for various values of Reynolds number $(\operatorname{Re}=0,50,100)$. It is clear that the magnitude of friction force decreases with increasing Reynolds number and it does not depend on Reynolds number at a certain value of flow rate. 


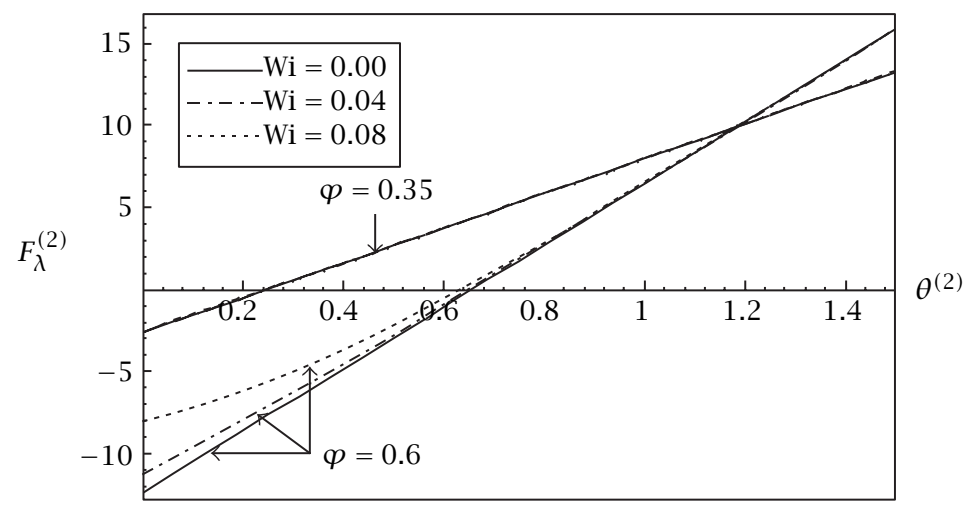

FIGURE 6.5. The friction force versus flow rate at $\mathrm{Re}=0.1, \delta=0.156$, and $\varphi=0.35,0.6$.

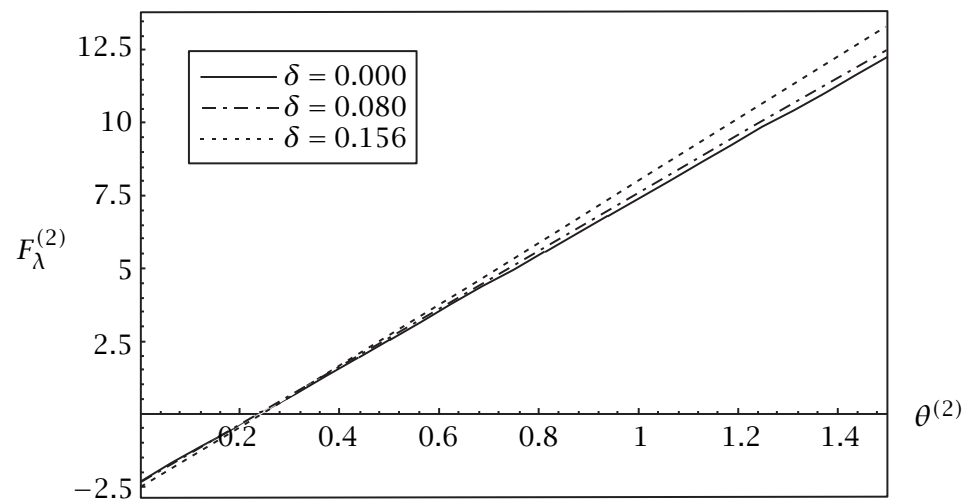

FIGURE 6.6. The friction force versus flow rate at $\mathrm{Re}=0.1, \mathrm{Wi}=0.08$, and $\varphi=0.35$.

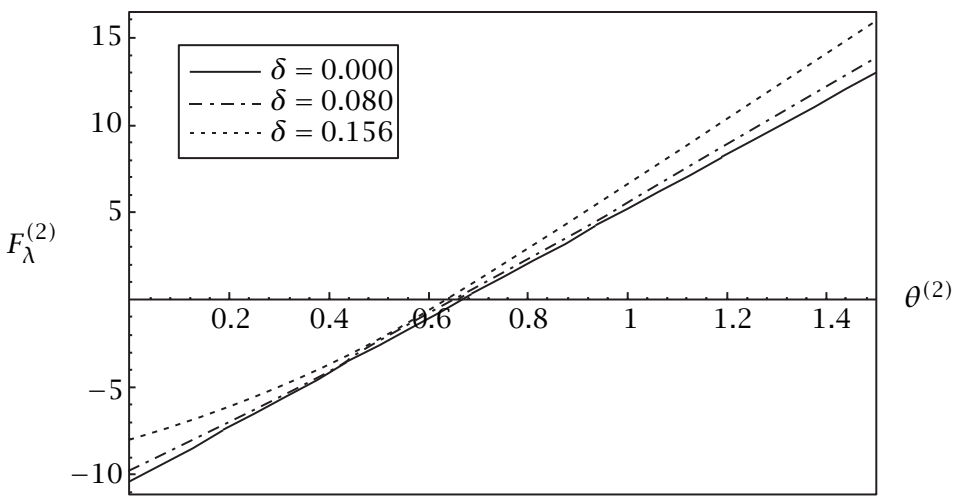

FIGURE 6.7. The friction force versus flow rate at $\mathrm{Re}=0.1, \mathrm{Wi}=0.08$, and $\varphi=0.6$. 


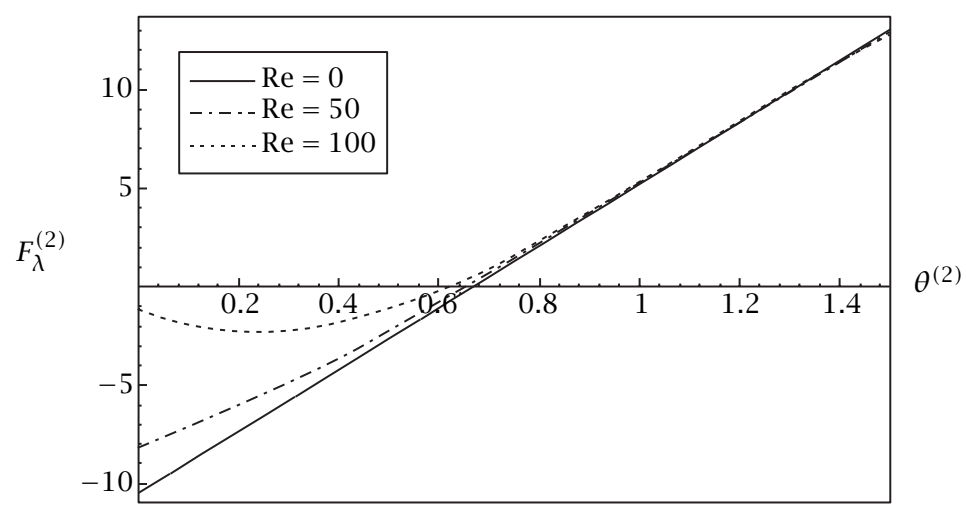

FIGURE 6.8. The friction force versus flow rate at $\mathrm{Wi}=0, \delta=0.02$, and $\varphi=0.6$.

\section{REFERENCES}

[1] G. Bohme and R. Friedrich, Peristaltic flow of viscoelastic liquids, J. Fluid Mech. 128 (1983), 109-122. Zbl 515.76131.

[2] A. M. El Misery, E. F. Elshehawey, and A. A. Hakeem, Peristaltic motion of an incompressible generalized fluid in a planar channel, J. Phys. Soc. Japan 65 (1996), 501-506.

[3] E. F. Elshehawey, A. M. El Misery, and A. A. Hakeem, Peristaltic motion of a generalized Newtonian fluid in a non-uniform channel, J. Phys. Soc. Japan 67 (1998), 434-440.

[4] E. F. Elshehawey and K. S. Mekheimer, Couple-stresses in peristaltic transport of fluids, J. Phys. D: Appl. Phys. 27 (1994), 1163-1170.

[5] M. Y. Jaffrin and A. H. Shapiro, Peristaltic pumping, Annual Review of Fluid Mechanics, vol. 3, Annual Reviews, 1971, pp. 13-36.

[6] T. W. Latham, Fluid motion in a peristaltic pumping, Master's thesis, Massachusetts, MIT Cambridge, 1966.

[7] R. K. Rathy, An Introduction to Fluid Dynamics, Oxford and IBH Publishing Co., New Delhi, 1976. MR 56\#17393.

[8] A. H. Shapiro, M. Y. Jaffrin, and S. L. Weinberg, Peristaltic pumping with long wavelength at low Reynolds number, J. Fluid Mech. 37 (1969), 799-825.

[9] A. M. Siddiqui and W. H. Schwarz, Peristaltic motion of a third-order fluid in a planner channel, Rheol. Acta 32 (1993), 47-56.

[10] L. M. Srivastava, Peristaltic transport of a couple-stress fluid, Rheol. Acta 25 (1986), 638641.

[11] L. M. Srivastava and V. P. Srivastava, Peristaltic transport of blood. Casson model II, J. Biomech. 17 (1984), 821-829.

[12] _ Peristaltic transport of a non-Newtonian fluid: applications to vas defernes and small intestine, Ann. Biomech. Engin. 13 (1985), 137-153.

[13] _ Peristaltic transport of a power-law fluid: application to the reproductive tract, Rheol. Acta 27 (1988), 428-433.

Elsayed F. Elshehawey: Department of Mathematics, Faculty of Education, Ain Shams University, Heliopolis, CAIro, Egypt

Ayman M. F. Sobh: Department of Mathematics, College of Education, Gaza, P.O. Box 4051, GAZA STRIP, PALESTINIAN NATIONAL AUTHORITY

E-mail address: a_sobh@hotmai 1 .com 


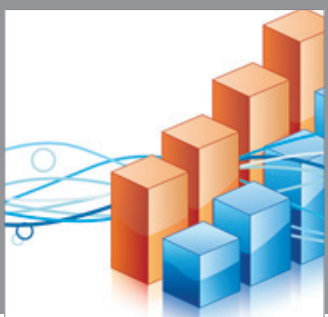

Advances in

Operations Research

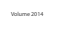

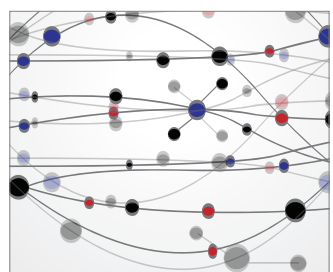

\section{The Scientific} World Journal
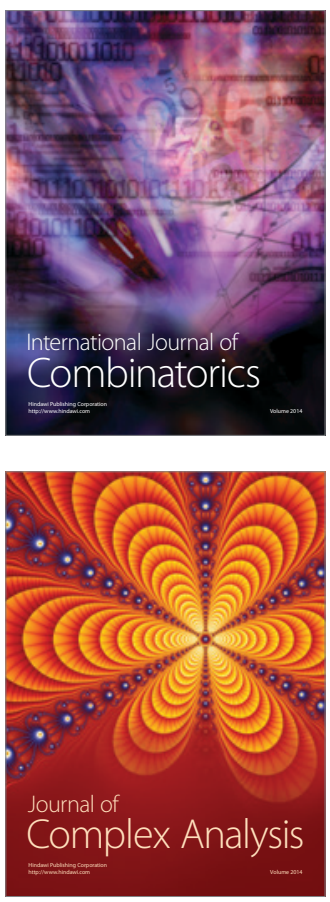

International Journal of

Mathematics and

Mathematical

Sciences
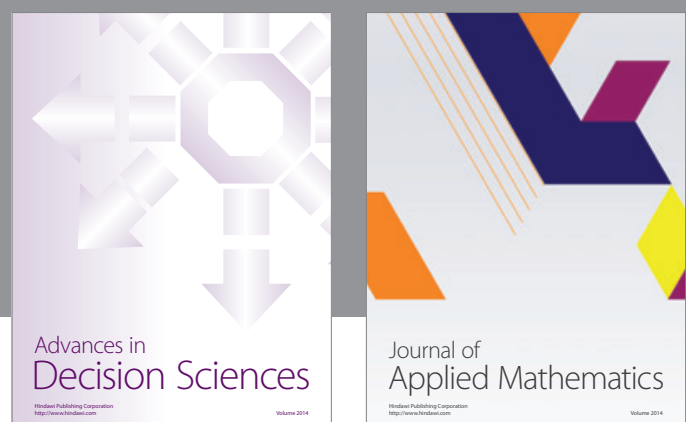

Journal of

Applied Mathematics
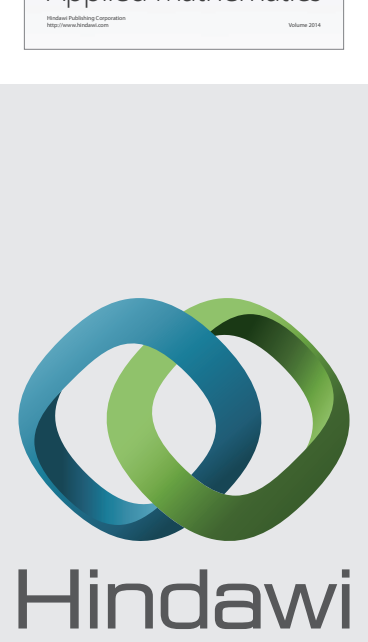

Submit your manuscripts at http://www.hindawi.com
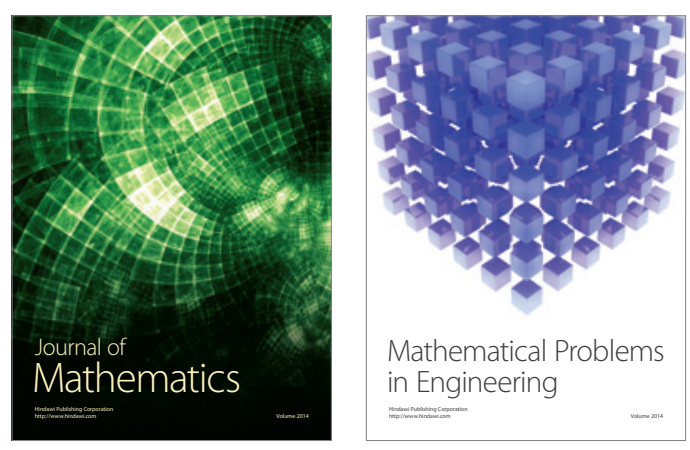

Mathematical Problems in Engineering
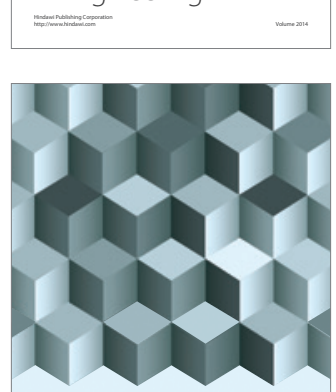

Journal of

Function Spaces
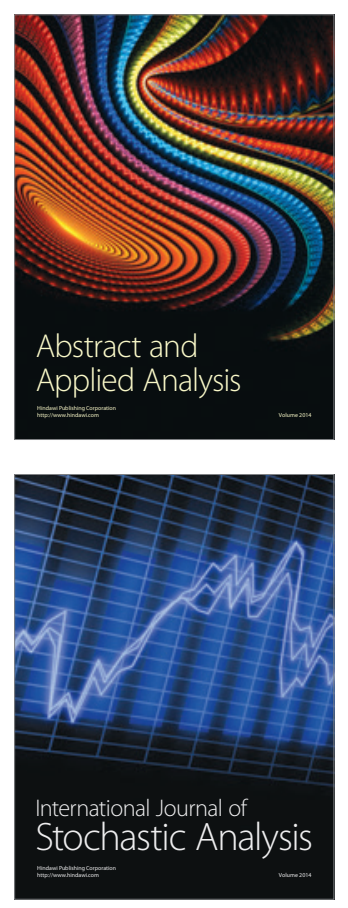

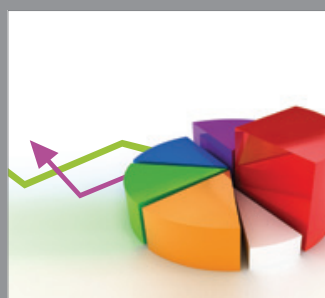

ournal of

Probability and Statistics

Promensencen
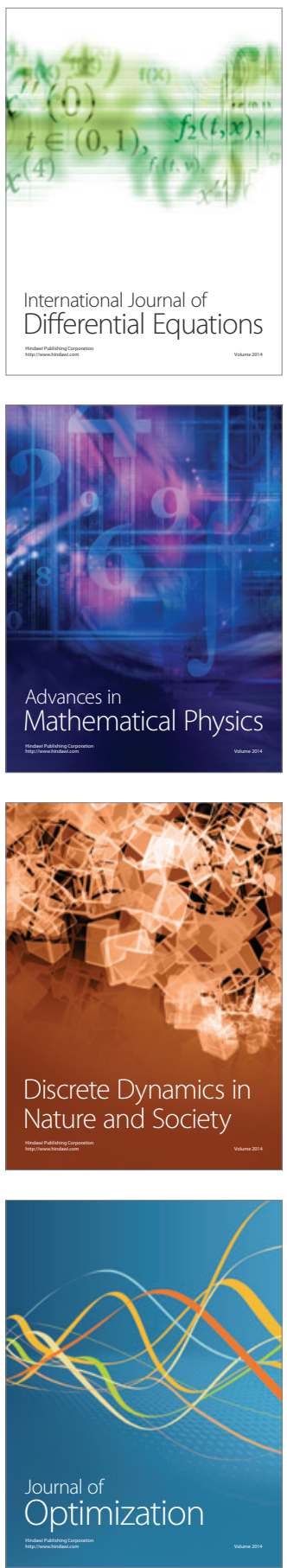